\title{
Solid State Physics; Magnetic Phenomena Progress Report
}

l January 1972 - 31 December 1972

R. Bowers and R. H, Silsbee

AEC Contract No. AT $(11-1)-3150$

Laboratory of Atomic and Solid State Physics Cornel1 University Ithaca, New York 14850

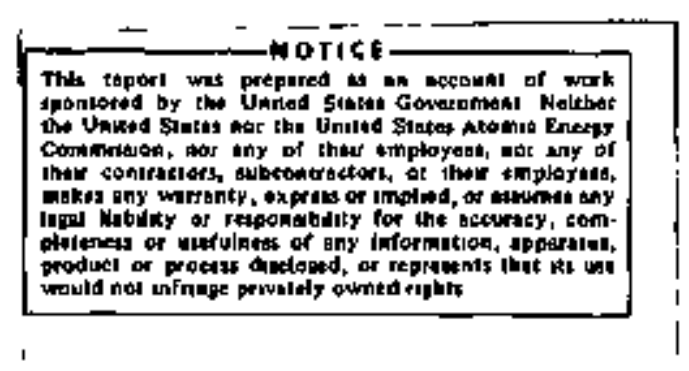




\section{DISCLAIMER}

This report was prepared as an account of work sponsored by an agency of the United States Government. Neither the United States Government nor any agency Thereot, nor any of their employees, makes any warranty, express or implied, or assumes any legal liability or responsibility for the accuracy, completeness, or usefulness of any information, apparatus, product, or process disclosed, or represents that its use would not infringe privately owned rights. Reference herein to any specific commercial product, process, or service by trade name, trademark, manufacturer, or otherwise does not necessarily constitute or imply its endorsement, recommendation, or favoring by the United States Government or any agency thereof. The views and opinions of authors expressed herein do not necessarlly state or reflect those of the United States Government or any agency thereof. 


\section{DISCLAIMER}

Portions of this document may be illegible in electronic image products. Images are produced from the best available original document. 


\section{Electron Spin Resonance \\ (Scope I) \\ Co-Investigator: R. H. Silsbee}

The two principal areas of interest using the technique of microwave resonance remain the dynamic properties of orientable defects at low (liquid helium) temperatures and the electron spin resonance (ESR) of dilute metal alloy systems.

Dr, Hans Bill, a Visiting Fellow from the University of Geneva, has worked intensively for the past year on the properties of the paramagnetic $0^{-}$ion incorporated as a substitutional Impurity in $\mathrm{CaF}_{2}$. Although not at first right an "orientable defect", the presence of the orbital electronic degeneracy of the $\mathrm{O}^{-}$ion and the high synutetry of the $\mathrm{F}^{-}$site in $\mathrm{CaF}_{2}$ imply that the system is susceptible to the Jahn-Teller effect. There are in fact three possible equivalent static Jahn-Teller distortions, corresponding to distortions along each of the three (100) directions, and the dynamics of the reorientation among these three distortions are intimately connected to the reorientation dynamics of molecular defects. The experimental work involved a careful study of line position, line shape and width, and saturation properties of the $0^{-}$(ESR)as a function of temperature and applied uniaxial stress. The results are 
consistently interpreted in detail in terms of a phenomenological model or spin hamiltonian involving both the properties (electronic state and spin orbit coupling) of the free $0^{-}$ion and its interaction (strain coupling coefficients) with the host crystal. A simple model for the Jahn-Teller problem seems adequate to give rather a good picture of the dynamical behavior. A new mechanism involving simultaneous electron spin flip and distortional reorientation is identified and discussed. The results are in preparation for publication.

our attempts to extend the influence of host isotopic constitution upon the reorientation rate of the $\mathrm{O}_{2}{ }^{-}$impurity in the alkali. halides have not been successful. "Raschke carried out apparently successful experiments on $\mathrm{kCl}$ enriched with $\mathrm{K}^{41}$ for his thesis project. He then tried to extend these results to determine the influence of a changed $c 1$ isotopic constitution, but was unable to obtain satisfactorily reproductble results. We currently do not have sufficient resources in terms of manpower to pursue this problem.

The dynamics of $\mathrm{N}_{2}^{-}$molecular reorientation are closely related to those of the $\mathrm{O}_{2}{ }^{-}$except that the $\mathrm{N}_{2}{ }^{-}$ESR spectrum shows a hyperfine splitting resulting from the $\mathrm{N}^{14}$ nuclear moment and the $\mathrm{N}_{2}{ }^{-}$shows the unexpected behavior that the $90^{\circ}$ reorlentation processes are more rapid than the $60^{\circ}$ processes. These two observations imply that the system $\mathrm{KL}: \mathrm{N}_{2}^{-}$should be 
ideal for the confirmation of the prediction by Sussman that the reorientation rate will depend upon the symmetry of the nuclear spin state of the molecule, We have observed such a dependence, the puzzling result being that the observed effect is substantially smaller than anticipated. Since this implies that reorientation mechanisms other than the conventional Sussman mechanism are operative, we are currently examining other possibilities for the reorientation process in order to develop a consistent picture with which to describe the data.

The remaining system of interest in the area of orientable defects is the strong tunneling systen, $\mathrm{KCl}: \mathrm{Li}$, in which we had planned to extend the paraelectxic (PER) measurements to pumped liquid $\mathrm{He}^{3}\left(\sim .3^{\circ} \mathrm{K}\right)$ during the past year. The motivation for this extension was the expectation that most of the mechanisms for spectral diffusion and cross relaxation would be strongly suppressed in the regime hw $>\mathrm{kT}$ and that the microwave saturation of the resonance would be dramatically altered at the low temperature. Although a number of experimental problems associated with this extension to lower temperature were solved, the rather slow experimental progress stimulated a more careful look at the ideas behind the experiment to be sure that the extension to liquid $\mathrm{He}^{3}$ temperature would really be worthwhile. A theoretical model has been developed to describe the fundamental excitations of the coupled system, lithium plus phonons, 
at low temperature (kT $<\mathrm{hw}_{\mathrm{o}}$ ) which allows a quantitative prediction of the 1ifetimes of these excitations and which suggests that pulse rather than steady state saturation experiments will be more informative. The development of these experiments by Mr, Vitko is in progress.

In the area of ESR in metals, this year has been devoted to the analysis of data obtained by Siebext and Dodds and some supplementary experiments both on ESR and D. C. resistivity. as a function of temperature on these same alloys of aluminum with gadolinium, erbium and lutetfum (nominal) impurities. The ESR of A1:Gd shows strong evidence of bottleneck effects and it will probably be important to perform a variety of further experiments before attempting a detailed interpretation of the results, Since we had available these alloys which are rather difficult ones to prepare, it seened worthwhlle to obtain data on the resistivity versus temperature simply to verify that no grossiy anomalous behavior was apparent. Indeed no Kondo minImum is observed, and both the magnitude of the residual resistivity and of the deviations from Matthiessen's rule are comparable with the size of these effects due to other inpurities.

The erbium ESR analysis is simpler than that for the gadolinium because the $g$ value of the erbium resonance (about 6) is markedly different from that of the conduction electrons, and bottleneck effects are weak or non-existent. Our ability 
to measure both the raxe earth xesonance and the conduction spin resonance allows a detailed analysis of the results. The exchange interaction between the rare earth and conduction electron moments results in shifts and broadening of both resonances and intercomparison of the results allows a stringent consistency check on the interpretation. In addition to giving the exchange interaction constant, the temperature dependence of the shift of the conduction electron line induced by the polarization of the rare earth spins allows a determination of the temperature dependence of the expectation $\langle\mathrm{Sz}\rangle$ of the rare earth spin. This dependence may then be interpreted in terms of crystal field. models to give the splittings of the lowest level of the free rare earth lon by the crystal field of the host crystal.

A sample nominally doped with lutetium was included in these Elrst experiments as a control since it is expected to have a ful1 $4 \mathrm{f}$ she11 and no magnetic moment. The surprising observation of a temperature dependent line width and $g$ shift of the conduction spin resonance raised some question as to the starting material which was supplied to us and, Indeed, a cheraical analysis has verified that the rare earth was in fact thulium and not lutetium as advertised by the supplier. The trivalent thulium ion is a non Kramers system (two $4 \mathrm{f}$ holes) and one does not expect to observe ESR for this system, but 
the analysis of the conduction spin line shift and width again, as in the case of erbium, allows a determination of crystal field splittings of the thalium ion. These results are in the process of analysis. 
Magnetic Phenomena and Electronic Structure

$\underline{\text { Scope }}$ II

Co-Investigator: $R$. Bowers

Our research can be grouped into three interacting areas: (a) studies of transport effects in certain metals with emphasis on the elucidation of certain scattering mechanisms, (b) exploitation of high frequency heiicon wave propagation characteristics to determine the high-field Hall coefficient, and (c) studies of the electromagnetic generation of acoustic waves in metals. The primary motivation for area (a) comes from our belief that after two decades of concentrated study of the band structure of metals, we are entering a period where it should be possible to make major progress on understanding the influence of different scattering processes on the various transport properties of meta1s. New experfments utilizing electrical and thermal resistivity measurements and the radio frequency sizeeffect promise to give useful new information about scattering processes. Much of our experimental work has centered on potassium and tungsten because of the simple band structure of the former and the very long mean free paths that are available in the latter. Area (b) is of interest in a more spectific sense than (a); the specimen geomezry most favorable 
for high frequency helicon measurements can eliminate many specimen-related problems when one is working with soft and/or reactive metals. Thus, in potassium it is possible to make more reliable and accurate measurements than can be done using other techniques. Although relatively little work has been done this past year in area (c), we continue to view th as a very fruitful research area. Our interest in developing a fundamental understanding of electromagnetic generation is amplified because of its potential importance from an applied point of view. Progress on completed and continuing projects is outlined below.

‥ Our study of the zero-field thermal resistance of potassium has been completed. The detailed comparison that we were able to make between theory and experiment demonstrated that it was worthwhile solving the numerous experimental problems we encountered in working with potassfum. The following abstract of a paper to be published in February gives a good description of this work.

The thermal resistivity of single and polycrysta1line potassium specimens having residual resistance ratios between 195 and 6500 has been measured between 1.5 and $15 \mathrm{~K}$. It is found that theoretical calculations based on semiclassical ideas are in reasonably good agreement with the experimental data, particularly for the more impure specimens at low temperatures. The role of unklapp scattering is clearly evident. The magnitude of the calculated thermal resistivity is very near the experimental result for impure specimens 
but the calculated values are too high for the pure specimens. It is shown that when impurity scattering is dominant, a variational calculation of the electron'phonon thermal resistance should be quite accurate. In pure specimens the calculations are not expected to be nearly as good; reasons for this are discussed; At high temperatures, above 6 to $8 \mathrm{k}$, the theoretica1 temperature dependence is in considerable disagreement with the experimental results. In specimens of low and intermediate purity the contribution of lattice thermal conduction can be appreciable and should be taken into account when making comparions with theory.

Thermal resistance measurements have been made on many specimens of potassium in magnetic fields up to $18 \mathrm{kG}$. The experimental results are not in agreement with semiclassical theory. In addition, they are inconsistent with a thermal resistance deduced from electrical resistance measurements using the Wiedemann-Franz law. In contrast to the electrical case, the thermal magnetoresistance is very large. To better understand these puzzling results, we plan to extend this work to higher fields.

Recently, we have completed measurement of the highfleld Hall coefficient in single-crystal potassium in fields up to $100 \mathrm{kG}$. This work is described by the following abstract of a paper that has been submitted for publication.

The magnetic field dependence and magnitude of the high-field Hall coeffictent of potassitm have been determined experimentally by studying the propagation characteristics of high-frequency helicon waves. Between 20 and $100 \mathrm{kG}$ no significant $( \pm 0.5 \%)$ field dependence is observed in the Hall coefficient for two different crystalline orientations. Absolute measurements yleld values which are, depending on the 
orientation, 4 or $8 \%$ larger in absolute value than the free-electron Hall coefficient. Accuracy of the magnitude measurements is estimated at $\pm 2 \%$. Sample thicknesses are measured in situ using a transducerless ultrasonic technique. The temperature dependence of the Hall coefficient has also been measured. To within $0.05 \%$ no change in that quantity is observed upon cooling the sample from $4.2 \mathrm{~K}$ to $2.2 \mathrm{~K}$. The experimental results are discussed in the light of recent theories on the magnetoresistance of potassium which predict a decreasing Hall. coefficient with increasing magnetic field.

A very interesting series of experiments concerned with the influence of specimen surface condition on the lowtemperature dc electron transport properties of singlecrystal tungsten rods has been completed. The following genera1 results are observed. Surface condition is found to affect the magnitude of both the electrical and thermal resistivities. The temperature dependence of the electrical resistivity is observed to change reversibly with surface condition, while that of the thermal resistivity does not change in the range of our observations. The Lorenz number varies also with surface condition and for samples of sufficient purity with highly polished surfaces its value extrapolated to zero temperature tends to a value somewhat below the value $L_{o}=2.44 \times 10^{-8} \mathrm{~W}-\Omega / \mathrm{K}^{2}$. In a longitudinal magnetic field, the Lorenz number increases to $L_{o}$, which indicates unambiguously that this deviation from $\mathrm{L}_{o}$ is a surface effect. We are unable at present to explain this effect. To investigate this problem further we are currently 
seeking a means of studying in detail scattering of Fermi. energy electrons from carefully prepared surfaces.

Significant progress has been made on a project to measure the anisotropy of the electron mean-free path in potassium by means of the radio frequency size effect (RFSE), Because of the difficulty of fabricating the thin, single-crystal plates with smooth and parallel faces that ultimately are necessary for the experiment, mach effort has been devoted to designing the experiment so that maximum information can be obtained from each specimen. This requires that the rf excltation of surface currents be cylindrically symetric with respect to the normal of the sample surface and that the static magnetic field be rotatable about this same axis while maintaining a spectfic tilt angle out of the sample plane. The first requirement has been met in a recently completed and tested rf transmission apparatus by using spiral coils for both excitation and detection of the rf surface currents. The latter requirement has been satisfied by the construction of a specially designed superconducting magnet system and power supp1y, which together produce a field of up to $.8 \mathrm{~T}$ having any desired spatial orientation.

Calculations of the magnetothermoelectric power of aluminum have been completed. The results of these calculations have been compared with measurements of the' magnetothermoelectric 
power of aluminum made by R. S. Averback; the following is the abstract of a recent_paper_on this work.

Measurements of the low-temperature magnetothermoelectric power of aluminum and a number of aluminum alloys show that the electron-diffusion component $S_{d}$ increases and then saturates with increasing magnetic field. The difference $\Delta \mathrm{S}_{\mathrm{d}}$ between high- and zerofield values of $s_{d}$ is observed to be independent of the type of impurify in the aluminum and has the value $(2.2 \pm 0.2) \mathrm{T} \times 10^{-8} \mathrm{v} / \mathrm{K}$. These observations are Interpreted in terms of a realistic semiclassical analysis of the themopower. This treatment gives an excellent account of the qualitative behavior of the thermopower in a magnetic field but yields a value of $\Delta \mathrm{S}_{\mathrm{d}}$ which is $30 \%$ lower than the observed value. Possible reasons for this discrepancy are discussed.

No experjmental work has been done in the past year on electronagnetic generation of acoustic waves in zero magnetic field. A calculation by a summer research student of the influence of a rough surface on electromagnetic generation failed to show any significant effect below $100 \mathrm{MHz}$.

A short project aimed at demonstrating the feasibility of using electromagnetic detection to observe acoustic enission in conducting solids has been completed. The following is an_abstract of work to appear in print shortly.

The acoustic emission resulting from the martensitic phase transformation in a gold-47.5at.\% cadmium alloy has been observed using a purely electromagnetic means to detect the acoustic signals. This detection method eliminates transducer-related resonances which usually make a determination of the frequency spectrum associated with acoustic emission very difficult. Both the temporal and frequency response of individual acoustic emission avents have been determined. For the small specimen used in this 
investigation the frequency response is dominated by acoustic cavity resonance effects in the specimen. This work shows that electromagnetic detection of acoustic emission may be especially useful when attempting to deternine the frequency spectrum of various souxces of acoustic emission. 


\section{$\underline{\text { Personne1 }}$}

Dr. Curt Raschke has joined the research staff of the Addressograph-Multigraph Corporation in Cleveland, ohio.

Dr. Hans Bill has returned as an assistant professor to the University of Geneva.

\section{Publications}

1. "Generation and Detection of $55 \mathrm{GHz}$ Phonons Using Paxaelectric Resonance of $\mathrm{KCl}: \mathrm{Li}, " \mathrm{~T} . \mathrm{R}$. Larson and R. H. Silsbee, CH-3150-83, Phys, Rev. B 5, 3927 (1972).

2. "Electron Paramagnetic Resonance of Color Centers,". R. H. Silsbee, a chapter in the review volune "Electron Paramagnetic Resonance", edited by S. Geschwind, Plenum Press, New York (1972).

3. "Electronagnetic Detection of Acoustic Emission from a Maztensitic Transformation," B. W. Maxfield and R. Cochran, Coo-3150-1 and to be published, Materials Evaluation, February 1973.

4. "The Influence of Magnetoacoustic 0scillations on Electromagnetic Excitation of Shear Acoustic Waves," M. R. Gaerttner and B. W. Maxfield, c00-3150-4 and Phys. Rev. Letters 29, 654 (1972). 
5. "Thermal Resistivity of Potassium Between 1.5 and $15 \mathrm{~K}$," R. S. Newrock and B. W. Maxfield, co0-3150-5 and to be published, Physical Review, February 1973.

6. "The Thermal. Resistivity and Thermal Magnetoresistivity of Potassitm," R. S. Newrock and B. W. Maxfield, Coo-3150-6 and to be published in the Proceedings of the 13th International Low Tempexature Conference, Boulder, Colorado, August 21-25, 1972.

7. "hall Coefficient of Potassiun Determined by a Helicon Transmission Method," D. E. Chimenti and B. W. Maxfield, co0-3150-9 and submitted to Physical Review.

8. "Low Termerature Magnetothermoelectric Power of Aluminum," R. S. Averback and D. K. Wagner, $C 00-3150-3$ and to be published, Solid State Conmunications.

9. "Effect of Surface Condition on the Lorenz Number and Other Electron Transport Properties of Size-Limited SingleCrystal Tungsten Rods," D. R. Baer and D. K. Wagner, CoO3150-7 and to be published in the Proceedings of the 13th International Low Temperature Conference, Boulder, Colorado, August 21-25, 1972. 


\section{$\underline{\text { Talks }}$}

"Electronic Transport Properties of Size Limited Tungsten Wires," D. R. Baer, Bull. Am. Phys. Soc., Series II, 17, 255 (1972).

"Magnetic Field Dependence of the Hall Coefficient of Potassiun," D. E. Chimenti and B. W. Maxfield, Bull. Am. Phys. Soc., Series II, 17, 279 (1972).

"Effect of Surface Condition on the Lorenz Number and other Electron Transport Properties of Size-Linited Single-Crystal Tungsten Rods," D. R. Baer and D. K. Wagner, 13th International Conference on Low Temperature Physics, Boulder, Colorado, August $21-25,1972$.

"Thermal Resistivity and Thermal Magnetoresistivity of Potassium," R. S. Newrock and B. W. Maxfield, 13th Internationa1 Conference on Low Temperature Physics, Boulder, Colorado, August 21-25, 1972 .

"Thermal and Magnetothermal Resistance of Potassium," R. S. Newrock and B. W. Maxfield, Bull. Am, Phys. Soc., Series II, 17, 256 (1972). 